\title{
Creating Pictures in Quick Response Codes
}

\author{
Jeremy C. Creasey \\ J\&FC Consulting Ltd \\ Great Oaks, Back Road, Catbrook, \\ Monmouthshire NP16 6NA, UK \\ jeremyc.creasey@gmail.com
}

\section{INTRODUCTION}

The Quick Response (QR) Code is a machine readable, two-dimensional barcode used to encode information attached to objects. They are found in product identification, advertising and other areas where metadata must be labelled concisely and unambiguously. QR codes are quickly decoded by machine, having in-built resilience to damage, obscuration or misalignment. They are generated through an internationally standardised procedure (ISO/IEC18004 2006) that converts simple text, a URL or executable commands into a square matrix of dots or modules.

This paper investigates the use of QR codes as an artistic form and considers if it is possible to create a bona fide symbol that is also recognisable by the human eye. The QR code must be generated with strict adherence to the ISO standard, not just incorporating a pasted-in image that is ignored due to error correction.

\section{QR CODES AS ART}

Many artists have incorporated the matrix form into their work (Blake 2013, Haase 2014, Falcon 2012). Error correction within the encoding algorithm allows pasting of graphics into the barcode and alterations to module sharpness, comparative reflectance and colour without compromising the ability to decode the symbol. However, this paper concentrates on producing an artistic QR code with a "pure", adulterated matrix using only modules.

The encoding procedure is designed to produce reliable QR Codes. Data mask patterns are applied to the light and dark modules to create a wellbalanced matrix which is evaluated to avoid "undesirable features" such as too many dark modules in the entire symbol.

This means that valid machine-readable codes usually look like a set of pseudo-random dots to the human eye, except for the regular, square-shaped function patterns used for alignment and timing. The encoded region of the symbol combines both data and error correcting codewords in interleaved blocks. There are several challenges therefore in generating an eye-recognisable pattern in the matrix whilst adhering to the ISO rules. Figure 1 shows a basic face shape (middle-right of symbol) in a decipherable, version 4-H QR code.

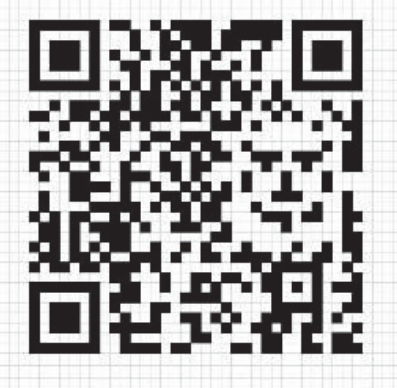

Figure 1: Shape of a face inside a bona fide QR code

\section{SIMPLIFYING THE CHALLENGE}

This paper describes the analysis of suitable QR code formatting modes and techniques to reverse engineer the seven step encoding procedure to obtain symbols that can be interpreted simultaneously by machine and human. Variations in matrix size, data masks and error detection modes will be explored and the results illustrated with more aesthetic examples than Figure 1.

\section{REFERENCES}

ISO/IEC18004 (2006) Information technology Automatic identification and data capture techniques - QR Code 2005 bar code symbology specification. ISO Copyright Office, Switzerland.

Blake, S. (2013) Barcode Art. Available at: http://www.barcodeart.com/artwork/index.html (Accessed: 1 January 2014). 
Haase, F. (2014) QR Art. Available at:

http://www.qr-art.com/index.html

(Accessed: 1 January 2014).

Falcon, A. (2012) 40 Gorgeous QR Code Artworks

That Rock. Available at:

http://www.hongkiat.com/blog/qr-code-artworks/

(Accessed: 1 January 2014). 